\title{
A CONTRIBUIÇÃO DA TEORIA COGNITIVA DE VYGOTSKY NO ENSINO DA GESTÃO DE PROJETOS NO CONTEXTO DA ENGENHARIA DE PRODUÇÃO
}

Mônica Holanda Santos ${ }^{a}$ - monica.holanda.santos@outlook.com

Rubens Alves Dias ${ }^{b}$ - rubens.alves@unesp.br

Arminda Eugenia Marques Campos ${ }^{a}$ - arminda.campos@unesp.br

Universidade Estadual Paulista - UNESP, Faculdade de Engenharia, Guaratinguetá

(a)Departamento de Produção Mecânica, (b) Departamento de Engenharia Elétrica

Avenida Ariberto Pereira da Cunha

12516410 - Guaratinguetá - São Paulo

Resumo: Em razão das mudanças políticas e econômicas em âmbito mundial e da alteração dos comportamentos sociais e de aprendizagem, faz-se necessária para acompanhar o atual cenário e as necessidades do mercado de trabalho a adequação dos conteúdos estudados nas universidades. Nesse contexto, identifica-se a responsabilidade dos docentes na escolha de teorias educacionais como ferramentas voltadas aos processos de ensino e aprendizagem, de forma que o aluno domine os significados dos conteúdos ensinados e apresente condições de colocá-los em prática, na resolução de problemas nos diversos campos de atuação profissional. A adequação do ensino às necessidades referidas se apresenta na aplicação de teorias educacionais. O presente trabalho baseia-se na teoria sociointeracionista de Vygotsky como proposta de referencial teórico educacional no ensino da engenharia de produção no Brasil, tendo como projeto piloto a disciplina de Gestão de Projetos. Por meio de uma revisão bibliográfica foi possível identificar o estado da arte da teoria e suas respectivas aplicações no ensino, mostrando um significativo potencial de agregação de valor ao ensino da engenharia como um todo.

Palavras-chave: Engenharia de produção. Educação em ensino superior. Gestão de projetos. Teoria cognitiva. Vygotsky.

\section{INTRODUÇÃO}

No Brasil, a procura por cursos de engenharia tem aumentado significativamente com a taxa média de crescimento de 14,6\% ao ano. Dentre várias áreas que a engenharia pode ofertar, destaca-se a Engenharia de Produção, a qual está em quinto lugar no ranking dos cursos que mais formaram até o ano de dois mil e dezessete (INEP, 2017). O perfil do curso é multidisciplinar, pois recorre aos conhecimentos especializados da matemática, física, ciências humanas e sociais, conjuntamente com os princípios e métodos de análise e projeto da engenharia para fins didáticos (ABEPRO, 2019).

Na classificação de áreas de atuação e pesquisa proposta pela Associação Brasileira de Engenharia de Produção, a gestão de projetos é uma das subáreas da área de Engenharia 
Organizacional (ABEPRO, 2019). Tem importância, no entanto, em outras áreas, como Ergonomia, Engenharia do Produto e Engenharia da Qualidade, entre outras. Constitui-se de uma área de ampla utilidade e aplicação, que exige liderança, organização, trabalho em equipe e comunicação para entender e delegar ideias e funções. De acordo com Scheidmandel (2018) a gestão de projetos se constitui como uma grande filosofia técnica para ser aplicada em empreendimentos de qualquer escopo.

Em razão das mudanças econômicas em âmbito mundial e da alteração dos comportamentos sociais e de aprendizagem, faz-se necessária a adequação dos conteúdos estudados nas universidades para acompanhar o atual cenário e as necessidades do mercado de trabalho e da sociedade. Visando preparar e qualificar os alunos, órgãos responsáveis e conferências exercem pressão para que instituições se adequem aos padrões mais contemporâneos.

Nesse contexto, identifica-se a responsabilidade dos docentes na escolha de teorias educacionais como ferramentas voltadas aos processos ensino aprendizagem, de forma que o aluno domine os significados dos conteúdos ensinados e consiga colocá-los em prática, para resolução de problemas nos diversos campos de atuação. Observa-se que a comunicação dentro da sala de aula influencia a construção do significado dos conceitos apresentados, tendo o docente um papel primordial (KIEMER; GRÖSCHNER, PEHMER, SEIDEL, 2015; NAIDOO; LIKWAMBE, 2018).

Ressalta-se que a formação do engenheiro é um bacharelado e alguns engenheiros que se tornam docentes desconhecem as teorias de aprendizagem, próprias da licenciatura (RAMMAZZINA FILHO; BATISTA; LORENCINI, 2014). Situações como esta podem ocasionar falta de preparo para o planejamento de aulas, priorizando gostos pessoais nas escolhas pedagógicas interferindo negativamente na potencialidade do ensino e na avaliação da aprendizagem dos alunos. O ensino contextualizado na engenharia com conteúdo didático de estudo de casos auxilia os docentes nas percepções necessárias à melhoria dos processos ensino e aprendizagem. Para Mazzioni (2014) e Hwang (2013) a forma como o docente planeja suas atividades de sala de aula determina interesse dos alunos e contribui com o andamento das atividades educacionais. Conteúdos didáticos no ensino da engenharia, de preferência com estudos de caso (ensino contextualizado), auxiliariam aos mesmos a terem percepções necessárias para uma melhora no processo.

Percebe-se por uma revisão de literatura, que não há número significativo de trabalhos que exploraram as Teorias de Aprendizagem dentro da engenharia, cujas pesquisas e métodos desenvolvidos ao serem aplicados têm o potencial de contribuir para a melhoria das atividades educacionais. Para Ertmer e Newbay (2013) as Teorias de Aprendizagem são uma fonte de estratégias, táticas e técnicas de instrução verificadas. Tais teorias são divididas em quatro principais enfoques teóricos: comportamentalista/behaviorista, cognitivista, construtivista/humanista e, sendo uma abordagem contemporânea, a representacionista (MILNER; TEMPLIN; CZERNIAK, 2011; MOREIRA, 2013).

Reconhece-se que a falta de conteúdo ofertado para enriquecer os planejamentos e formações dos docentes pode ocasionar dificuldades de ordem didático-pedagógicas para os docentes, com implicações negativas nos ambientes de ensino. Neste contexto educacional em que a Engenharia de Produção como curso de grande procura e de formação rebuscada carece de estratégias educacionais para a potencialização do ensino da engenharia, por intermédio de boas práticas de ensino, pergunta-se: Há pesquisas, com as contribuições de Vygotsky, aplicadas ao ensino da gestão de projetos no contexto da engenharia de produção no Brasil? Como objetivo geral procura-se identificar o estado da arte da teoria cognitiva e se ela está aplicada ao ensino da gestão de projetos no contexto da engenharia de produção no Brasil. 


\section{REVISÃO DE LITERATURA}

A análise por meio de uma revisão de literatura, de diversos documentos relacionados às teorias de aprendizagem, mostra que a maioria dos artigos refere-se ao ensino fundamental e médio e nas áreas similares a engenharia foram aplicados de forma benéfica. Por exemplo, a teoria de Vygotsky auxilia o aluno na criação de significados para símbolos, como os matemáticos, os quais o seu cognitivo absorve o conteúdo explicado e o reproduz resolvendo situações idênticas no cotidiano.

\subsection{Estado da arte}

Utilizou-se a base de dados do Scopus a fim de coletar os artigos relacionados ao tema proposto por meio de um filtro de palavras-chaves e combinações das mesmas, visando identificar trabalhos e autores. A pesquisa foi aberta a todos os anos para saber a quantidade de documentos publicados. Os tipos de documentos filtrados foram artigos e documentos de revisão (review) e os parâmetros foram: Título do artigo, abstract e palavras-chaves, conforme Quadro 1.

Quadro 1: Comparação de palavras

\begin{tabular}{|c|c|c|c|}
\hline Base de dados & Palavras-chave & Número de documentos & Categoria \\
\hline Scopus & $\begin{array}{l}\text { ("Vygotsky" "vygostki" "vigotsky" "vigotski") AND ( "social interaction theory" } \\
\text { "social interacionism theory" } \\
\text { "social interaction" } \\
\text { "social interacionism " } \\
\text { "socio-historical theory" } \\
\text { "socio historical theory" } \\
\text { "socio cultural theory" } \\
\text { "socio cultural" } \\
\text { "social - interaction theory" } \\
\text { "social - interacionism theory") }\end{array}$ & 183 & $\begin{array}{l}\text { Artigo e } \\
\text { documentos } \\
\text { de revisão } \\
\text { (Review) }\end{array}$ \\
\hline Scopus & $\begin{array}{l}\text { ("Vygotsky" "vygostki" "vigotsky" "vigotski") AND ( "social interaction theory" } \\
\text { "social interacionism theory" } \\
\text { "social interaction" } \\
\text { "social interacionism " } \\
\text { "socio-historical theory" } \\
\text { "socio historical theory" } \\
\text { "socio cultural theory" } \\
\text { "socio cultural" } \\
\text { "social - interaction theory" } \\
\text { "social - interacionism theory") AND ("Case Study") }\end{array}$ & 10 & $\begin{array}{l}\text { Artigo e } \\
\text { documentos } \\
\text { de revisão } \\
\text { (Review) }\end{array}$ \\
\hline Scopus & $\begin{array}{l}\text { ("Vygotsky" "vygostki" "vigotsky" "vigotski") AND ( "social interaction theory" } \\
\text { "social interacionism theory" } \\
\text { "social interaction" } \\
\text { "social interacionism " } \\
\text { "socio-historical theory" } \\
\text { "socio historical theory" } \\
\text { "socio cultural theory" } \\
\text { "socio cultural" } \\
\text { "social - interaction theory" } \\
\text { "social - interacionism theory") AND ("engineering education") }\end{array}$ & 2 & $\begin{array}{l}\text { Artigo e } \\
\text { documentos } \\
\text { de revisão } \\
\text { (Review) }\end{array}$ \\
\hline
\end{tabular}

Fonte: Elaborado pela autora, baseado em Scopus

As combinações foram colocadas em aspas, para encontrar artigos e artigos de revisão (reviews) que contenham o uso da teoria sem perder o sentido da busca, e evitando assim mostrar resultados parciais que contenham somente algumas palavras buscadas. Utilizaram-se 
várias formas e abreviações ao escrever as palavras tornando possível a busca pelo maior número de artigos possíveis relacionados ao tema com resultado favorável.

Os documentos referentes à teoria de Vygotsky mostram que ela é significativa e abordada em diversas áreas ao longo dos anos tendo seus primeiros artigos publicados na década de 1980 e atualmente utilizada em baixo número de publicações por ano, com apenas dois artigos na área de educação em engenharia, com a contribuição de (Rowell, 2002) e (Apsara, 2016).

Dos 183 artigos analisados, apenas dez abordavam a teoria e/ou aplicações do autor cognitivista no ensino escolar com estudo de caso aplicado como o material principal da pesquisa. Os demais artigos eram voltados para as áreas de meditação, autismo, amamentação, psiquiatria, marketing e nutrição, usando a teoria de Vygotsky para distinção de classes sociais e convivência. Percebe-se que, pela importância do tema, necessidades atuais e publicações, há uma lacuna de pesquisa.

No ranking dos países com maior número de documentos, destacam-se os Estados Unidos da América em primeiro lugar com 28 documentos, a maioria com alto índice de citação, o Reino Unido em segundo lugar, com 19, e o Brasil em terceiro lugar, com sete documentos. Percebe-se que os números estão bem distantes de serem considerados uma concorrência acirrada, porém, ressalta-se a importância do reconhecimento dos pesquisadores brasileiros por estarem em um dos melhores lugares do ranking, e pela aplicabilidade da teoria. Deve-se aproveitar a oportunidade e fomentar com mais pesquisas para ganhar espaço no cenário nacional.

\subsection{Referencial teórico}

Lazarowitz, (1994) aplicou a teoria de Vygotsky, com 73 alunos de um grupo experimental e 47 de outro grupo individual, durante cinco semanas com pré e pós testes. Concluiu-se que os alunos do grupo experimental se destacavam na maioria dos critérios: resultados acadêmicos (normativa e objetiva), domínio afetivo, quantidade de amigos, maior autoestima e envolvimento durante as aulas. Não houve diferença de grupos nos critérios: coesão, cooperação, competição, e atitudes referentes ao assunto aprendido. Após alguns anos, Ahmadian (2014) teve uma aplicação de pesquisa parecida, utilizando a teoria interacionista de Vygotsky e o diálogo colaborativo para analisar os vocabulários nos critérios de quantidade e conhecimento de 64 alunos iranianos divididos em dois grupos, um experimental e outro individual, com base em seis tarefas com três estágios sendo elas antes, durante e depois da experiência. Concluiu-se que o grupo que trabalhou no coletivo aumentando drasticamente o vocabulário, permitindo que os alunos construíssem suas zonas de desenvolvimento em colaboração com o compartilhamento do conhecimento de outros alunos.

Ambas as pesquisas indicaram que os alunos desenvolveram suas zonas de desenvolvimento melhor quando estavam em contato com outros alunos, do que quando isolados. A troca de conhecimento com um mediador mais capaz para orientar faz com que haja diversidade de significados. No contexto de aprendizagem com um agente mais capaz mediando o conhecimento, destaca-se o trabalho de Rowell (2002), no qual foram examinadas cooperação, colaboração e concretização de ideias, sob a perspectiva da teoria sociointeracionista de Vygotsky e sua zona de desenvolvimento proximal - ZDP (conceito que descreve a "distância" entre o conhecimento existente e o desejável), com a participação de dois alunos do ensino fundamental, na tarefa tecnológica da construção de um robô. Os alunos foram identificados como iniciadores de ideias; em certo momento um aluno assumiu o posto de mediador, permitindo que o outro entendesse e acompanhasse sua forma de pensar para cumprir a tarefa, explicitando dessa forma a importância da interação social. 
Os professores, como um todo, se preocupam e perdem mais tempo com o gerenciamento de estudantes do que com as maneiras pelas quais as ideias são trocadas e desenvolvidas (ROWELL, 2002). Sugere-se atentar ao funcionamento da sala de aula, já que em uma atividade tecnológica pode-se observar diversos acontecimentos importantes. Acredita-se que não há um roteiro para se solucionar problemas, cada um é como se fosse novo; o que existe são estratégias educacionais mais adequadas, conforme a situação, que favorecem o desenvolvimento da forma de pensar do indivíduo (LIBÂNEO, 2018). Destaca-se que embora tenha aparecido no filtro de estudos aplicados a engenharia, o artigo não é aplicado neste contexto.

Ben-ari (2000) em sua pesquisa já dizia ter dificuldades nas diversidades contidas nos grupos de estudantes e afirma que o aumento da heterogeneidade é uma tendência mundial nas salas de aula, ocasionando diversidade cultural, social e econômica, afetando o desempenho escolar e tornando-se um desafio para os professores proporem atividades que todos possam compreender. O estudo foi aplicado a 1017 alunos de oito a onze anos de idade, em 36 salas de aula, em grupos heterogêneos, com respectivas tarefas de acordo com a matriz curricular regular. Obtiveram o apoio de professores e supervisores treinados anteriormente para realizar as anotações e tarefas. Os resultados confirmaram que existiu uma relação positiva entre a interação social na tarefa e o fato de os professores incentivarem verbalmente, afetando indiretamente a mudança cognitiva. Nota-se que o papel do professor como agente mais capaz é essencial no processo de ensino aprendizagem, nos próximos estudos pode-se observar os impactos dos mesmos e seus respectivos resultados com as ferramentas mais adequadas aos alunos estudados.

Khoza (2018) focou seu estudo nas ferramentas pedagógicas de três professores, dos quais $80 \%$ dos ensinamentos se davam por meio de palestras, para 209 alunos do último ano do ensino superior de biologia. Concluiu-se que por meio da narrativa de histórias do conteúdo estudado, acionou-se uma interação cognitiva em que uma imagem se processou na mente gerando significados. Atividades com instruções claras despertaram nos alunos interação individual com o conteúdo abordado, absorção do conteúdo e condução do pensamento ao meio social mediado para geração da interação e engajamento do aprendizado. Comprovou-se a teoria de Vygotsky que diz que a aprendizagem é a internalização da interação social, desencadeada por trocas sociais, como gestos, palavras, conversas, escrita e imagens visuais.

Yang (2018) apoiou-se no pensamento de Vygotsky afirmando que a emoção precisa ser analisada dentro do sistema unificado de conceitos, assim como outras funções psicológicas, e aplicou em um estudo de caso com 31 voluntários do programa de formação de professores antes e depois de terem a experiências na sala de aula. Por meio da combinação de métodos qualitativos e quantitativos compreendeu se as ligações das emoções e seus vínculos cognitivos, confirmando que as emoções fazem parte de um sistema complexo de ideias, conceitos e imagens, inter-relacionadas ao cognitivo que permeiam o processo de solução de problemas.

Destaca-se que há a necessidade dos professores elaborarem ferramentas de apoio adequadas para ao processo de aprendizagem, geração de resultados e sentimentos positivos, para crença no autodesenvolvimento, aumento da criatividade e inovação no ensino. Tem-se como sugestão futura, um modelo de aprendizagem que atenda as emoções de alunos e professores, capaz de lidar com as emoções durante a vida profissional.

Albert (2000) tem como base principal de sua pesquisa, a teoria de Vygotsky de que o desenvolvimento não pode ser separado dos contextos sociais, ou da linguagem oral e escrita. Aplicou-se pesquisa a sete alunos do ensino médio, explorando-se a relação entre procedimentos e estratégias de resolução de problemas, para compreensão de conceitos 
matemáticos. Em entrevistas para compreender o nível de conhecimento dos alunos e suas ideias, escritas como em um diálogo interno que segundo Vygotsky trata-se de um processo de autorregulagem que mostra que o aluno está em um nível superior de aprendizado. Os alunos aprimoram seus conhecimentos ao comunicarem o aprendido, por meio de práticas de solução de problemas com discussões e escrita de ideias, interesses e experiências que os ajudou a acompanhar seus pensamentos e soluções.

Muller (2016) focou seu estudo na teoria de Vygotsky que defende que as emoções são vistas como parte integrante da cognição e aprendizagem, e aplicou seu estudo em doze salas de aula das escolas primárias e juniores da Suíça. Participaram doze professores e 232 alunos (de onze a dezesseis anos). Realizou experiências de diversidade cultural, com imagens dos conteúdos estudados, experiências de verbalização do próprio conhecimento e expressão do conhecimento das emoções do outro aluno em palavras.

A escolha das imagens visou despertar nos alunos emoções específicas como alegria e tristeza, facilitando a interação e aprendizagem do significado de outros colegas de classe contribuindo para construção de conhecimento, acontecimento que Vygotsky chama de emoções inteligentes.

O estudo de Apsara (2016) é o que mais se assemelha à atual pesquisa proposta, embora não tenha sido aplicada. $\mathrm{O}$ autor identificou que os projetos de construção de navios, tidos como inovadores, não incluíam um entendimento do ambiente operacional e dos desafios práticos do cotidiano tornando por vezes inviável o projeto. Durante o período de formação os alunos tinham pouco contato com profissionais que se formaram e que trabalhavam colocando em prática o que aprenderam. Montou-se um quadro teórico com as etapas de implantação das três teorias complementares aplicadas: a aprendizagem baseada em problemas, a aprendizagem liderada por pares e a zona de desenvolvimento de Vygotsky.

\section{A GESTÃO DE PROJETOS NUMA ABORDAGEM SOCIOINTERACIONISTA}

Percebe-se que uma formação de qualidade no ensino superior causa impactos positivos no mercado de trabalho, uma vez que transforma o aluno em um profissional capacitado para resolver problemas reais do cotidiano organizacional. Uma das necessidades para que este processo aconteça, é fazer com que o aluno tenha estímulos cognitivos, para que ele coloque o que aprendeu na teoria em prática.

Sendo a área de engenharia necessária à sociedade na resolutividade de problemas em todos os segmentos, a engenharia, seja civil, naval, elétrica, ou qualquer um de seus outros ramos forma profissionais que lidam diretamente com cálculos e teorias que trabalham o cognitivo. Para que estes saberes sejam cheios de significados e possam ser empregados na vida profissional do aluno, as teorias de aprendizagem devem fortalecer e elucidar esses processos de aprendizagem.

A formação em gestão de projetos é propícia ao uso de tais teorias, pois seu foco está voltado a capacitar o indivíduo em aspectos de liderança, gerenciamento de tempo e de recursos, delegação de tarefas, proposição de escopo dos projetos, coordenação de pessoas e de atividades. O gestor de projetos precisa se comunicar com as pessoas, entender o prazo de cada processo da etapa do projeto e assim delegar funções de uma forma que todos entendam a sua responsabilidade particular dentro de cada projeto da organização.

$\mathrm{Na}$ abordagem proposta por Vygotsky o processo de aprendizagem acontece por meio da interação social, respeitando os contextos históricos, sociais e culturais. Ao utilizar as teorias de aprendizagem de Vygotsky em suas aulas, o docente consegue apresentar novos saberes aos alunos, trabalhando como mediador em um processo que respeita a bagagem 
cultural que este aluno leva consigo. Isso permite ao estudante de engenharia identificar possíveis áreas de aplicação das teorias ensinadas na prática do dia a dia.

A interação social é um meio eficaz para que os docentes entendam seus alunos e percebam dentro de suas potencialidades e necessidades a maneira que mais se adapta ao processo de aprendizagem, para que de maneira confortável e eficaz, essa teoria se torne realidade na vida profissional deste aluno, com um significado importante e de entendimento claro.

A disciplina de Gestão de Projetos tem um caráter multidisciplinar, que muitas vezes utiliza como apoio disciplinas anteriormente estudadas ou experiências concretas de projetos pessoais ou profissionais. As teorias ao serem aplicadas encontram na gestão de projetos um campo de atuação de mediação entre o que se aprendeu, o que o aluno leva consigo para a sala de aula, a partir de suas experiencias anteriores e a significação dos novos saberes passados a ele pelo docente.

Torna-se assim uma oportunidade de pesquisa e um desafio aplicar a teoria de Vygotsky no contexto da formação em Engenharia de Produção, também marcadamente multidisciplinar. Desafio este que mostra a partir das teorias, que pode ser seguido em várias outras áreas da engenharia por contribuir para os processos educacionais nas áreas interdisciplinares e multidisciplinares, além de favorecer a interação entre aluno e docente ao respeitar aspectos históricos, culturais e sociais de ambas as partes na mediação de novos saberes.

A partir da literatura, em sua maior parte voltada aos ensinos fundamental e médio, tendo trabalhos dedicados ao ensino superior em menor quantidade, a teoria sociointeracionista tem significativo potencial de agregar valor ao ensino da engenharia como um todo. Entretanto, pesquisar o referido referencial teórico educacional na disciplina Gestão de Projetos, devido à sua característica multidisciplinar e interdisciplinar, torna um interessante balão de ensaio para outras disciplinas.

\section{CONSIDERAÇÕES FINAIS}

A presente pesquisa, ora em desenvolvimento, supre as necessidades da área relacionada e apresenta contribuições acadêmicas significativas principalmente para o país que apresenta índices significativos de educação relacionados ao ranking internacional, estando entre os três melhores colocados podendo incentivar possíveis pesquisas futuras. Destaca-se também, a contribuição à sociedade, já que a formação do profissional afeta a qualidade do trabalho do engenheiro no mercado de trabalho.

A revisão de literatura foi capaz de identificar o atual estado da arte da teoria cognitiva, tanto no referencial teórico como no número de documentos. Considera-se que a diversidade de amostras, países, contextos organizacionais e idades dos alunos, contribuíram para a demonstração de que é possível aplicar e colher resultados positivos da teoria. Atente-se ao fato de que todos os estudos foram baseados nos pensamentos de Vygotsky e estruturados com métodos qualitativos e/ou quantitativos para que a coleta de dados fosse passível de identificação.

Como resposta à pergunta da presente pesquisa, relata-se que não há estudos de Vygotsky aplicados à engenharia de produção. $\mathrm{O}$ único artigo que apresenta uma proposta parecida é referente à engenharia naval. Deve-se, porém, destacar que os resultados obtidos desse artigo, foram significativos, demonstrando que a teoria cognitiva é aplicável na área de engenharia, podendo ser expandida, em particular, ao ensino da engenharia de produção.

Neste contexto, nota-se que para que tais impactos positivos ocorram, torna-se necessário ao professor preparo pessoal e profissional para utilizar a teoria cognitiva como referencial 
educacional. Domínio das emoções, autoconhecimento e conhecimento dos próprios limites e potenciais tornam-se características essenciais ao ato de lecionar, fomentando sentimentos positivos, aumentando a criatividade e a confiança no processo educacional.

Ressalta-se a importância do método de ensino no processo educacional para a absorção do conteúdo pelo aluno, gerando significado para que este consiga na prática solucionar problemas. Como visto nos resultados dos estudos da revisão de literatura, o aluno deve primeiro interiorizar individualmente o conteúdo, para depois contribuir socialmente com este conhecimento adquirido. O processo de compartilhar conhecimento torna as pessoas entendedoras das particularidades e cultura dos indivíduos, impactando no aumento e ampliação do vocabulário a partir do qual os signos passam a ter significados variados.

A aplicação das teorias de aprendizagem de Vygotsky nos processos educacionais no curso de Engenharia de Produção pretende fornecer ao docente ferramentas eficazes e eficientes aos engenheiros em formação. Ao utilizar tais teorias e então respeitar os referencias histórico-culturais e sociais do aluno, é possível ao docente, a partir do que se vive hoje apresentar e fazer-se entender e significar, pelo aluno teorias em aplicação hoje visando soluções de problemas não somente hoje, mas no exercício da profissão escolhida.

As vivências em sala de aula por ambas as partes tornam o professor mais que um detentor de conhecimentos, torna-o um mediador, capaz de aprimorar a forma de pensar dos alunos, com ações cognitivas de resolutividade e de transformação social e cultural na sociedade em que vive.

\section{Agradecimentos}

Este trabalho foi financiado pela Coordenação de Aperfeiçoamento de Pessoal de Nível Superior - Brasil (CAPES) - Código Financeiro 001.

\section{REFERÊNCIAS}

ABEPRO. Associação Brasileira de Engenharia de Produção. Profisssão. Disponível em: http://portal.abepro.org.br/a-profissao/. Acesso em: 02 abril 2020.

AHMADIAN, M.; TAJABADI, A. A case study showing how one young child represented issues concerned with attachment and separation in her spontaneous explorations.

International Journal of Applied Linguistics and English Literature, Buenos Aires, v.3(4), p. 38-45, 2014.

ALBERT, L. Outside in inside out: Seventh-Grade Students' Mathematical Thought Processes. Educational Studies in Mathematics, v. 41, n. 2, 2000.

APSARA, A.; MARGARETA, L.; STYHR, P.; HOSSEIN, E. Human-centred design knowledge into maritime engineering education; theoretical framework. Journal of Engineering Education, Australasian, 21:2, 49-60, 2016.

BEN-ARI, R.; KEDEM-FRIEDRICH, P. Restructuring heterogeneous classes for cognitive development: Social interactive perspective. Instructional Science, v. 28, n. 2, p. 153-167, 2000 . 
"Os desafios para formar hoje o engenheiro do amanhã"

ERTMER, P. A.; NEWBY, T. J. Behaviorism, Cognitivism, Constructivism: Comparing Critical Features From an Instructional Design Perspective. Performance Improvement Quarterly, v. 26, n. 2, p. 43-71, 2013.

FRANCISCHETTI, I. Active Learning Methodologies: An Experience for Faculty Training at Medical Education. Creative Education, v. 5, n. 21, p. 1882, 2014.

HWANG, G. J. et al. A learning style perspective to investigate the necessity of developing adaptive learning systems. Semantic Sholar, v. 16, n. 2, p. 188-197, 2013.

INEP. Instituto Nacional de Estudos e Pesquisas. Censo do Ensino Superior. Disponível em: https://www.gov.br/inep/pt-br. Acesso em: 02 abril 2020.

KIEMER, K., GRÖSCHNER, A., PEHMER, A.-K., SEIDEL, T. Effects of a classroom discourse intervention on teachers' practice and students' motivation to learn mathematics and science. Learning and Instruction, v.35, p. 94-103, 2015.

KHOZA, C.; NYAMUPANGEDENGU, E. Prompts Used by Biology Lecturers in Large Lecture Group Settings to Promote Student Interaction. African Journal of Research in Mathematics, Science and Technology Education, v.22, p. 386-395, 2018.

LAZAROWITZ, R.; BAIRD, H. Learning science in a cooperative setting: Academic achievement and affective outcomes. Journal of Research in Science Teaching, v. 31(10), p. 1121-1131, 1994.

LIBÂNEO, José Carlos. Didática. São Paulo: Cortez, 263p., 2018.

MAZZIONI, S. As estratégias utilizadas no processo de ensino-aprendizagem: concepções de alunos e professores de ciências contábeis. Revista Eletrônica de Administração e Turismo, v. 2, n. 1, p. 93-109, 2013.

MULLER, N. Development and Materiality at School: a Cultural-Historical Approach. Springer Science Business Media, New York, 2016.

NAIDOO, J.; LIKWAMBE, B. Exploring the Nature of Dialogue within South African Preservice Teachers' Calculus Lecture Rooms. African Journal of Research in Mathematics, Science and Technology Education, 2018.

RAMMAZZINA, F.; BATISTA, I.; LORENCINI, A. Formação de professores de engenharia - desafios e perspectivas. In: Simpósio de ensino de ciência e tecnologia. Anais eletrônicos. Ponta Grossa, p. 1-10, 2014.

ROWELL, P.M. Peer Interactions in Shared Technological Activity: A Study of Participation. International Journal of Technology and Design Education, v.12, p.1-22 ,2002. 
"Os desafios para formar hoje o engenheiro do amanhã"

SCHEIDMANDEL, Nilo Alberto et al. Gestão de projetos na Engenharia de Produção: uma visão acadêmica. Passo Fundo: Editora Universidade de Passo Fundo, p.146, 2018.

YANG, S; LIN, W. Effects of online culturally responsive pedagogy: Collaborative learning between college tutors and indigenous high school students. Journal of Research in

Education Sciences, v. 60, p. 223-253, 2018.

\title{
THE CONTRIBUTION OF VYGOTSKY'S COGNITIVE THEORY IN TEACHING PROJECT MANAGEMENT IN THE CONTEXT OF PRODUCTION ENGINEERING
}

\begin{abstract}
Due to political and economic changes worldwide and changes in social and learning behaviors, it is necessary to adapt the content studied in universities to the current scenario and the needs of the labor market. In this context, the responsibility of teachers in the choice of educational theories is identified as tools aimed at the teaching-learning process, so that the student can master the meanings of the contents taught and present conditions to put them into practice, in solving problems in the different professional fields. The adequacy of teaching to the referred needs is presented in the application of educational theories. The present work is based on Vygotsky's sociointeractionist theory as a proposal for a theoretical educational framework in the teaching of production engineering in Brazil, with the project management discipline as a pilot project. Through a bibliographic review it was possible to identify the state of the art of the theory and its respective applications in teaching, showing a significant potential to add value to the teaching of engineering as a whole.
\end{abstract}

Keywords: Production engineering, Higher education, Project management, Cognitive theory, Vgotsky. 